\title{
Downlink Steered Space-Time Spreading Assisted Generalised Multicarrier DS-CDMA Using Sphere-Packing-Aided Multilevel Coding
}

\author{
Mohammed El-Hajjar, Ronald Y. S. Tee, Hu Bin, Lie-Liang Yang and Lajos Hanzo \\ School of Electronics and Computer Science, University of Southampton, SO17 1BJ, UK. \\ Email: $\{$ meh05r, ryst03r, 1ly, 1h\}@ecs.soton.ac.uk, \\ http://www-mobile.ecs.soton.ac.uk
}

\begin{abstract}
This paper presents a novel generalised Multi-Carrier Direct Sequence Code Division Multiple Access (MC DS-CDMA) system invoking smart antennas for improving the achievable performance in the downlink, as well as employing multi-dimensional Sphere Packing (SP) modulation for increasing the achievable diversity product. In this contribution, the MC DS-CDMA transmitter considered employs multiple Antenna Arrays (AA) and each of the AAs consists of several antenna elements. Furthermore, the proposed system employs both time- and frequency- (TF) domain spreading for extending the achievable capacity, when combined with a novel user-grouping technique for reducing the effects of Multiuser Interference (MUI). Moreover, in order to further enhance the system's performance, we invoke a MultiLevel Coding (MLC) scheme, whose component codes are determined using the so-called equivalent capacity based constituent-code rate-calculation procedure invoking a 4-dimensional bit-to-SP-symbol mapping scheme. Our results demonstrate an approximately $3.8 \mathrm{~dB} E_{b} / N_{0}$ gain over an identical throughput scheme dispensing with SP modulation at a BER of $10^{-5}$.
\end{abstract}

\section{INTRODUCTION}

Time-varying multipath fading, which imposes fundamental limitations on reliable wireless transmissions, has to be counteracted by sophisticated transceiver design. In [1], Alamouti proposed a simple transmit diversity scheme employing Space-Time Block Codes (STBC) [2], which was capable of achieving a substantial diversity gain. Inspired by the philosophy of STBC, Hochwald et al. [3] proposed the transmit diversity concept known as Space-Time Spreading (STS) for the downlink of Wideband Code Division Multiple Access (WCDMA) [4]. Furthermore, in recent years numerous research contributions have appeared on the topic of Multi-Carrier Direct Sequence Code Division Multiple Access (MC DS-CDMA), which constitutes an attractive scheme [4-6] based on a combination of DSCDMA and Orthogonal Frequency Division Multiplexing (OFDM). In [7,8], a generalised MC DS-CDMA scheme was proposed that includes the subclasses of both multitone and orthogonal MC DSCDMA as special cases. On the other hand, beamforming [9] constitutes an effective technique of reducing the multiple-access interference (MAI), where the antenna gain is increased in the direction of the desired user, whilst reducing the gain towards the interfering users. In [10], a hybrid downlink technique designed for achieving both transmit diversity and transmit beamforming was proposed for Direct Sequence Code Division Multiple Access (DSCDMA), which is referred to as Steered Space-Time Spreading (SSTS).

As a further advance, the concept of combining orthogonal transmit diversity designs with the principle of Sphere Packing (SP) modulation was introduced by $\mathrm{Su}$ et al. in [11], where it was demonstrated that the proposed SP-aided STBC system was capable of outperforming the conventional orthogonal design based STBC schemes of [1,2]. The SP-aided concatenated design of STBCs [11]

The financial support of Vodafone under the auspices of the Dorothy Hodgkin Postgraduate Award and that of the European Union within the Newcom and Pheonix projects, and the support of EPSRC, UK is gratefully acknowledged. was further developed by Alamri et al. [12] by invoking an iterative turbo receiver.

Multilevel coding (MLC) was proposed by Imai and Hirawaki [13] for protecting each bit of a non-binary symbol with the aid of different-rate binary codes. An attractive iterative multistage decoding (MSD) scheme was also proposed in [13] for attaining a high decoding performance at a low decoding complexity. In this MSD structure, the $i$ th bit constituting a specific protection class associated with the constituent code $C^{i}$ is decoded by the $i^{\text {th }}$ decoder, while simultaneously exploiting the a priori information obtained from the demodulator, before passing the information to the $(i+1)$ st protection level associated with the constituent code $C^{i+1}$. This MSD process is activated level by level at each different-rate component decoder, each of which constitutes a flexible component code that has numerous configurable parameters. The explicit advantage of having independently configurable parameters for the low-complexity component codes is that they may be appropriately adjusted for diverse applications. A beneficial technique, devised for determining each component MLC code's rate, was detailed by Wachsmann et al. in [14], where the design concept exploited the so-called chain-rule of mutual information introduced in [15].

The novelty and rationale of the proposed system can be summarised as follows:

1) We amalgamate the merits of SSTS and generalised MC DSCDMA for the sake of enhancing the system's performance by achieving both spatial diversity and beamforming gains. We demonstrate that the number of users supported is substantially increased by spreading in both the time and frequency $(T F)$ domain, where the Multi-User Interference (MUI) imposed can be minimised with the aid of a user-grouping technique.

2) We extend the concepts proposed in [14] for improving the design of our SSTS-aided MLC scheme invoking a 4-dimensional SP constellation. More explicitly, the equivalent capacity design [14] is further developed for determining the optimum LDPC constituent code rates of the SSTS-SP-MLC scheme in conjunction with various bit-to-SP-symbol mapping strategies in the 4-Dimensional SP space.

The rest of this paper is organised as follows. In Section II the philosophy of the downlink SSTS-aided generalised Multicarrier DSCDMA system is characterised. Section III describes how the SSTSaided generalised Multicarrier DS-CDMA system can be combined with SP modulation, followed by Section IV that describes how the proposed SSTS-SP-aided generalised Multicarrier DS-CDMA system can be combined with MLC. Finally, the attainable performance of these schemes is studied comparatively in Section V, followed by our conclusions in Section VI.

\section{SteEred Space-Time SpREading}

In [10] a hybrid technique designed for achieving both transmit diversity as well as beamforming referred to as SSTS was presented. 
The antenna architecture employed in Figure 1 for the SSTS scheme of [10] has $M=2$ distinct Antenna Arrays (AAs) spaced sufficiently far apart in order to achieve second-order transmit diversity. The $L$ number of beam-steering elements of each of the two AAs are spaced at a distance of $d=\lambda / 2$ for the sake of achieving beamforming. In [10] the SSTS scheme has been investigated in the context of DS-CDMA. By contrast, in this contribution we extend our study to the generalised MC DS-CDMA system of [7,8] employing $M=2$ transmit antenna arrays and a single receive antenna.

The system considered in this section is an evolutionary descendent of the generalised MC DS-CDMA scheme of [8] using $U \cdot V$ number of subcarriers. The transmitter schematic of the $k$ th user is shown in Figure 1, where a block of $2 U$ data symbols, each having a symbol duration of $T_{b}$, is Serial-to-Parallel $(\mathrm{S} / \mathrm{P})$ converted to $U$ parallel sub-blocks. The new symbol duration of each sub-stream becomes $T_{s}=U T_{b}$. Following the STS procedure described in [3], the transmitted signal is spread to $M=2$ transmit antennas with the aid of the $M=2$ orthogonal spreading codes of $\mathbf{c}_{k, 1}=\left[\begin{array}{ll}\mathbf{c}_{k} & \mathbf{c}_{k}\end{array}\right]$ and $\mathbf{c}_{k, 2}=\left[\begin{array}{ll}\mathbf{c}_{k} & -\mathbf{c}_{k}\end{array}\right], k=1,2, \ldots, K$ with $\mathbf{c}_{k}$ being the $k$ th user's spreading code. The $2 U$ outputs of the $U$ STS blocks modulate a group of subcarrier frequencies $\left\{f_{u, 1}, f_{u, 2}, \ldots, f_{u V}\right\}$ and then forwarded to the transmitter's beamformer. The symbol duration of the STS signal is $2 T_{s}$ and the discrete period of the orthogonal STS codes is $2 T_{s} / T_{c}=2 N_{e}$, where we have $N_{e}=T_{s} / T_{c}$ and $T_{c}$ represents the chip duration of the orthogonal STS codes. Finally, according to the $k$ th user's channel information, the $2 U V$ signals of the $k$ th user are weighted by the transmit weight vector $\mathbf{w}_{v, m}^{(k)}$ determined for the $v$ th subcarrier of the $k$ th user, which is generated for the $m$ th AA. As proposed for MC-CDMA schemes in [4], spreading in the F-domain may additionally be employed for the sake of increasing the diversity gain by exploiting the independent fading of the subcarriers in the F-domain. Hence in the generalised MC DSCDMA scheme advocated, the transmitted data stream can be spread in both the T-domain and the F-domain in order to support more users, while achieving a higher frequency diversity gain [7] by exploiting the independence of the time and frequency-domain fading. The resultant bandwidth is the same as that of the MC DS-CDMA scheme employing time-domain-only spreading, while the total number of users supported becomes $V \mathcal{K}_{\max }=V N_{e}$, which is $V$ times the number of users supported by the MC DS-CDMA scheme employing T-domain-only spreading. Let $\mathbf{c}^{\prime}{ }_{k}=\left\{c_{k}^{\prime}[0], c_{k}^{\prime}[1], \ldots, c_{k}^{\prime}[V-1]\right\}$ be the $k$ th user's orthogonal F-domain spreading code.

The general form of the $k$ th user's transmitted signal can be expressed as

$$
\begin{gathered}
\mathbf{s}_{k}(t)=\mathbf{s}_{k}\left(\tau+2 n T_{s}\right)=\left(\begin{array}{c}
\mathbf{s}_{k 1}\left(\tau+2 n T_{s}\right) \\
\mathbf{s}_{k 2}\left(\tau+2 n T_{s}\right)
\end{array}\right) \\
=\sqrt{\frac{2 P_{k}}{4 V L}}\left(\begin{array}{c}
\sum_{u=1}^{U} \sum_{v=1}^{V}\left[\mathbf{c}_{k 1} x_{k, u 1}+\mathbf{c}_{k 2} x_{k, u 2}^{*}\right] \mathbf{w}_{v, 0}^{k} c_{k}^{\prime}[v-1] \\
\sum_{u=1}^{U} \sum_{v=1}^{V}\left[\mathbf{c}_{k 1} x_{k, u 2}-\mathbf{c}_{k 2} x_{k, u 1}^{*}\right] \mathbf{w}_{v, 1}^{k} c_{k}^{\prime}[v-1]
\end{array}\right) \\
\times \quad \cos \left(2 \pi f_{u v} \tau+\phi_{k, u v}\right),
\end{gathered}
$$

where $0 \leq \tau<2 T_{s}, n=0,1, \ldots, P_{k} / V$ represents the transmitted power of each subcarrier, the factor $L$ in the denominator is due to beamforming and the factor $M M=4$ in the denominator suggests that the STS scheme using $M=2$ transmit antennas and $M=$ 2 orthogonal spreading codes distributes its power proportionally in space and time. Additionally, $\phi_{k, u v}$ represents the phase angle introduced in the carrier modulation process of user $k$ in the $u v$ th subcarrier and is uniformly distributed over $[0,2 \pi]$. The weight vector $\mathbf{w}_{v, m}^{(k)}, m=0,1$, represents the effect of the L-dimensional beamforming technique employed by the SSTS scheme.
The $L$-dimensional Spatio-Temporal (ST) Channel Impulse Response (CIR) vector between the $u v$ th subcarrier of the $k$ th user and the $m$ th AA can be expressed as $\mathbf{h}_{u v, m}^{(k)}(t)=\mathbf{a}_{u v, m}^{(k)}(t) \delta\left(t-\tau_{k}\right)=$ $\left[a_{u v, m 0}^{(k)}(t), \ldots, a_{u v, m(L-1)}^{(k)}(t)\right]^{T} \delta\left(t-\tau_{k}\right)$, where $\tau_{k}$ is the signal's delay, $a_{u v, m l}^{(k)}(t)$ is the CIR with respect to the $u v$ th subcarrier of the $k$ th user and the $l$ th element of the $m$ th AA. Based on the assumption that the array elements are separated by half a wavelength, we have

$$
\mathbf{a}_{u v, m}^{(k)}(t)=\alpha_{u v, m}^{(k)}(t) \mathbf{d}^{(k)},
$$

where $\alpha_{u v, m}^{(k)}(t)$ is a Rayleigh faded envelope, $\mathbf{d}^{(k)}=$ $\left[1, \exp \left(j\left[\pi \sin \left(\psi^{(k)}\right)\right]\right), \ldots, \exp \left(j\left[(L-1) \pi \sin \left(\psi^{(k)}\right)\right]\right)\right]^{T}$ and $\psi^{(k)}$ is the $k$ th user's direction of arrival (DOA).

Assuming that the $K$ users' signals expressed in the form of (1) are transmitted synchronously over the downlink Rayleigh fading channels, let $y_{u v, 1}$ and $y_{u v, 2}$ represent the first user's T-domain receiver correlator's output corresponding to the data transmitted on the $u v$ th subcarrier from the first and second antenna array, respectively. Hence, $y_{u v, 1}$ and $y_{u v, 2}$ can be expressed as:

$$
\begin{aligned}
& y_{u v, 1}=n_{u v, 1}+ \\
& \sum_{k=1}^{K^{\prime}} \sqrt{\frac{P_{k}}{V L}}\left[\left(\mathbf{w}_{v, 0}^{(k)}\right)^{\dagger} \mathbf{a}_{u v, 0}^{(1)} x_{k, u 1}+\left(\mathbf{w}_{v, 1}^{(k)}\right)^{\dagger} \mathbf{a}_{u v, 1}^{(1)} x_{k, u 2}\right] c_{k}^{\prime}[v-1] \\
& y_{u v, 2}=n_{u v, 2}+ \\
& \sum_{k=1}^{K^{\prime}} \sqrt{\frac{P_{k}}{V L}}\left[\left(\mathbf{w}_{v, 0}^{(k)}\right)^{\dagger} \mathbf{a}_{u v, 0}^{(1)} x_{k, u 2}^{*}-\left(\mathbf{w}_{v, 1}^{(k)}\right)^{\dagger} \mathbf{a}_{u v, 1}^{(1)} x_{k, u 1}^{*}\right] c_{k}^{\prime}[v-1]
\end{aligned}
$$

where $n_{u v, i}, i=1,2$ denotes the Additive White Gaussian Noise (AWGN), $\dagger$ denotes the conjugate transpose operation, and $K^{\prime}$ denotes the number of users sharing the same T-domain spreading code matrix with the desired user, where these otherwise inseparable users are differentiated by their unique F-domain codes.

By multiplying Equations (4) and (5) by the F-domain spreading code $c_{1}^{\prime}$ of user 1 and letting $\mathbf{w}_{m}^{k}=\mathbf{d}^{(k)}$, we have

$$
\begin{aligned}
z_{u v, 1} & =\sqrt{\frac{P_{k}}{V L}}\left[\alpha_{u v, 0}^{(1)} x_{1, u 1}+\alpha_{u v, 1}^{(1)} x_{1, u 2}\right] \\
& +\sum_{k=2}^{K^{\prime}} \sqrt{\frac{P_{k}}{V L}}\left[\left(\mathbf{w}_{v, 0}^{(k)}\right)^{\dagger} \mathbf{a}_{u v, 0}^{(1)} x_{k, u 1}+\left(\mathbf{w}_{v, 1}^{(k)}\right)^{\dagger} \mathbf{a}_{u v, 1}^{(1)} x_{k, u 2}\right] \\
& \times c_{k}^{\prime}[v-1] c_{1}^{\prime}[v-1]+n_{u v, 1}^{\prime} \\
z_{u v, 2} & =\sqrt{\frac{P_{k}}{V L}}\left[\alpha_{u v, 0}^{(1)} x_{1, u 2}^{*}-\alpha_{u v, 1}^{(1)} x_{1, u 1}^{*}\right] \\
& +\sum_{k=2}^{K^{\prime}} \sqrt{\frac{P_{k}}{V L}}\left[\left(\mathbf{w}_{v, 0}^{(k)}\right)^{\dagger} \mathbf{a}_{u v, 0}^{(1)} x_{k, u 2}^{*}-\left(\mathbf{w}_{v, 1}^{(k)}\right)^{\dagger} \mathbf{a}_{u v, 1}^{(1)} x_{k, u 1}^{*}\right] \\
& \times c_{k}^{\prime}[v-1] c_{1}^{\prime}[v-1]+n_{u v, 2}^{\prime},
\end{aligned}
$$

Finally, by invoking the STS receiver of [3], the signal received on the $u v$ th subcarrier can be decoded, assuming perfect knowledge of the fading parameters at the receiver side, to arrive at

$$
\tilde{x}_{u v, j}=\sqrt{\frac{P_{k}}{V L}}\left[\left\|\alpha_{u v, 0}^{(1)}\right\|^{2}+\left\|\alpha_{u v, 1}^{(1)}\right\|^{2}\right] x_{1, u j}+n_{u v, j}^{\prime}+i_{u v, j},
$$

where $j=0,1, i_{u v, j}$ denotes the MUI, which is inevitably imposed, since the orthogonality of the F-domain spreading codes cannot be retained over frequency-selective fading channels. However, the desired signal is not interfered by the transmitted signals of the users employing different orthogonal T-domain spreading codes, provided that synchronous downlink transmission of all the $K^{\prime} N_{e}$ user signals as well as flat-fading of each subcarrier are assumed. Only the users sharing their T-domain spreading code matrix with the desired user will impose MUI on the desired user. Therefore, the MUI can be reduced, if we carefully select the $\left(K^{\prime}-1\right)$ potentially interfering 


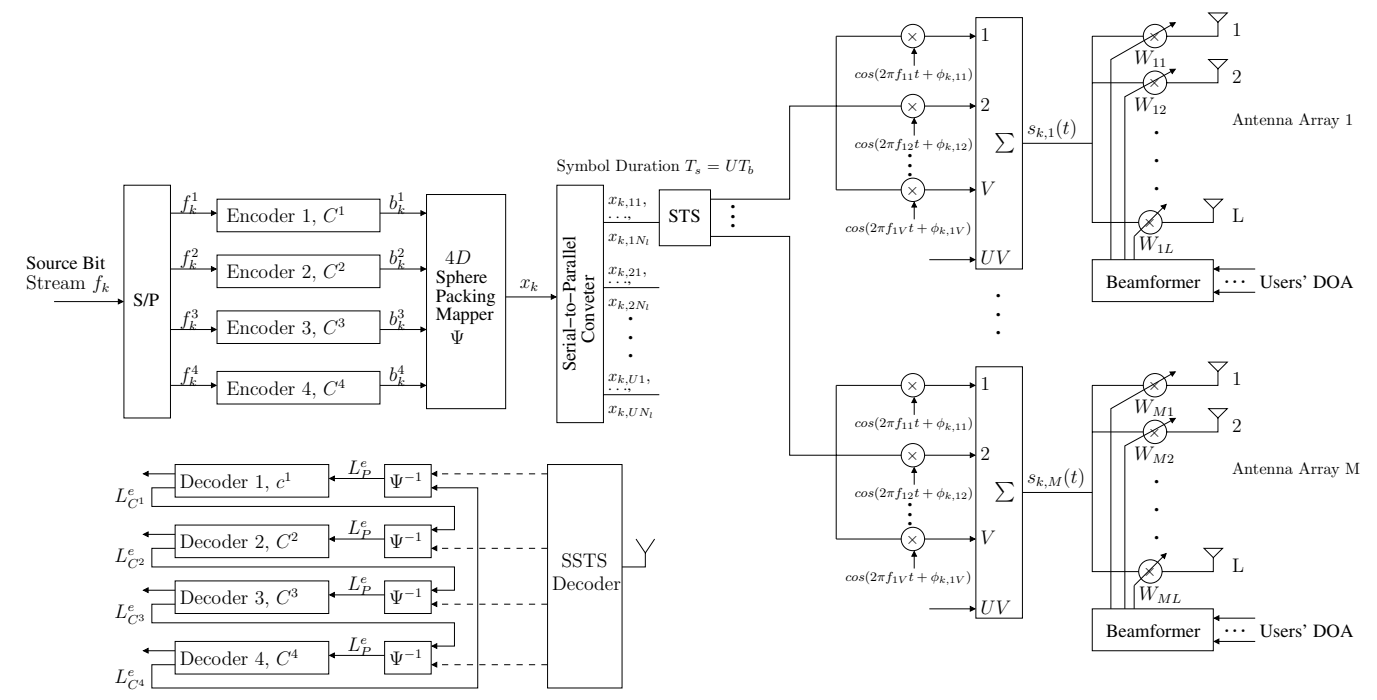

Fig. 1. The proposed system model.

users, namely those which have the lowest F-domain interference coefficient with respect to the desired user, from the entire set of all the $K^{\prime} N_{e}$ users. More explicitly, when selecting the specific users for the sake of sharing the same T-domain spreading code with the desired user, it must be ensured that their TF-domain interference remains low.

\section{SSTS DESIGN Using SPHERE PACKING Modulation}

It was shown in [11] that the so-called diversity product quantifying the achievable coding advantage ${ }^{1}$ of an orthogonal transmit diversity scheme is determined by the minimum Euclidean distance of the transmitted signal vectors. Hence, in order to maximise the achievable coding advantage, it was proposed in [11] to use SP schemes that maximise the minimum Euclidean distance of the transmitted signal vectors.

According to Equation (7), the decoded signals represent scaled versions of $x_{1, u 1}$ and $x_{1, u 2}$ corrupted by both the complex-valued AWGN and the MUI, when the system employs TF-domain spreading. This observation implies that the diversity product of the SSTSaided MC DS-CDMA system is determined by the minimum Euclidean distance of all legitimate vectors $\left(x_{1}, x_{2}\right)$, where the $u$ and $k$ indices are removed for simplicity. For the sake of generalising our treatment, let us assume that there are $L$ legitimate vectors $\left(x_{l, 1}, x_{l, 2}\right), l=0,1, \ldots, L-1$, where $L$ represents the number of sphere-packed modulated symbols. The transmitter, then, has to choose the modulated signal from these $L$ legitimate symbols, which have to be transmitted over the two AAs, where the throughput of the system is given by $\left(U \log _{2} L\right) / 2$ bits per channel use. In contrast to the independent transmitted signal design of (7), our aim is to design $x_{l, 1}$ and $x_{l, 2}$ jointly, so that they have the best minimum Euclidean distance from all other $(L-1)$ legitimate SP symbols, since this minimises the system's SP symbol error probability. Let $\left(a_{l, 1}, a_{l, 2}, a_{l, 3}, a_{l, 4}\right), l=0,1, \ldots, L-1$, be legitimate phasor points of the four-dimensional real-valued Euclidean space $\mathbb{R}^{4}$. Hence, $x_{l, 1}$ and $x_{l, 2}$ may be written as $\left\{x_{l, 1}, x_{l, 2}\right\}=\left\{a_{l, 1}+j a_{l, 2}, a_{l, 3}+j a_{l, 4}\right\}$.

In the four-dimensional real-valued Euclidean space $\mathbb{R}^{4}$, the lattice $\mathbb{D}_{4}$ is defined as a SP having the best minimum Euclidean distance from all other $(L-1)$ legitimate constellation points in $\mathbb{R}^{4}$ [16]. More specifically, $\mathbb{D}_{4}$ may be defined as a lattice that

\footnotetext{
${ }^{1}$ The diversity product or coding advantage was defined as the estimated gain over an uncoded system having the same diversity order as the coded system [11].
}

consists of all legitimate sphere-packed constellation points having integer coordinates $\left[\begin{array}{llll}a_{l, 1} & a_{l, 2} & a_{l, 3} & a_{l, 4}\end{array}\right]$ uniquely and unambiguously describing the legitimate combinations of the modulated symbols $x_{l, 1}$ and $x_{l, 2}$, but subject to the sphere packing constraint of $a_{l, 1}+a_{l, 2}+$ $a_{l, 3}+a_{l, 4}=\kappa$, where $\kappa$ is an even integer. Assuming that $S=$ $\left\{s^{l}=\left[a_{l, 1}, a_{l, 2}, a_{l, 3}, a_{l, 4}\right] \in \mathbb{R}^{4}: 0 \leq l \leq L-1\right\}$ constitutes a set of $L$ legitimate constellation points from the lattice $\mathbb{D}_{4}$ having a total energy of $E \triangleq \sum_{l=0}^{L-1}\left(\left|a_{l, 1}\right|^{2}+\left|a_{l, 2}\right|^{2}+\left|a_{l, 3}\right|^{2}+\left|a_{l, 4}\right|^{2}\right)$, and upon introducing the notation $C_{l}=\sqrt{\frac{2 L}{E}}\left(x_{l, 1}, x_{l, 2}\right), l=0,1, \ldots, L-1$, we have a set of complex constellation symbols, $\left\{C_{l}: 0 \leq l \leq L-1\right\}$, whose diversity product is determined by the minimum Euclidean distance of the set of $L$ legitimate constellation points in $\mathrm{S}$.

\section{SSTS-SP Aided Multi-LeVel Coding}

The schematic of the proposed SSTS-SP aided MLC (SSTS-SPMLC) arrangement is shown in Figure 1. The binary source bit stream $f_{k}$ of the $k$ th user is serial-to-parallel (S/P) converted at the transmitter. The four individual source bits, namely $f_{k}^{1}, f_{k}^{2}, f_{k}^{3}, f_{k}^{4}$, are protected by four different-rate MLC encoders, as seen in Figure 1. The output bits of encoder $C^{i}, i=1 \ldots 4$, having a total encoded frame length of $n$ bits are denoted as $b_{k}^{i}=b_{k 1}^{i}, b_{k 2}^{i}, \ldots, b_{k n}^{i}$.

Again, we employ LDPC component codes owing to their powerful error correcting capability, low complexity and flexible coding rates. The random nature of the parity check matrix construction of LDPC codes allows us to dispense with the employment of additional channel interleavers. Each LDPC codeword is decoded using the belief propagation algorithm [15]. The MLC encoded bit stream is then forwarded to the sphere packing modulator $\Psi$ of Figure 1 .

Figure 1 also shows the receiver structure of the system, where a SSTS decoder equipped with a single receive antenna is employed. The SSTS decoder forwards its complex-valued symbols to the SP-demodulator $\Psi^{-1}$ of Figure 1 and the resultant bits are then decoded at the different-protection LDPC decoders in an iterative MSD manner. The extrinsic LLRs $L_{P}^{e}$ of Figure 1 produced by the SP-demodulator are fed into the level-1 decoder of $C^{1}$, which then outputs a set of corresponding extrinsic LLRs $L_{C 1}^{e}$ to the demodulator. This LLR provides useful a priori information for the SP demodulator, where the LLRs gleaned from the previous protection level are updated. As the decoding process continues, each MSD level receives useful a priori LLRs from the previous MSD level, which can be exploited in the LDPC decoder. The next outer iteration seen in Figure 1 commences, when the LLR information of 


\begin{tabular}{|l||r|r|r|r|}
\hline Coding Rate & $R 1$ & $R 2$ & $R 3$ & $R 4$ \\
\hline SSTS-SP-MLC & $221 / 640$ & $193 / 640$ & $458 / 640$ & $408 / 640$ \\
\hline SSTS-QPSK-MLC & $425 / 640$ & $215 / 640$ & - & - \\
\hline
\end{tabular}

TABLE I

CODING RATES OF THE SSTS-SP-MLC AND SSTS-QPSK-MLC SCHEMES

\begin{tabular}{|l|r|}
\hline No. of Transmit AA M & 2 \\
\hline No. of Elements per Transmit AA L & 2 \\
\hline No. of Receiver Antennas & 1 \\
\hline Time-Domain Spreading Code & Walsh Code \\
\hline Time-Domain Spreading Factor & 4 \\
\hline Frequency-Domain Spreading Code & Walsh Code \\
\hline Frequency-Domain Spreading Factor & 4 \\
\hline Sphere Packing Modulation & L 16 \\
\hline Conventional Modulation & QPSK, Ungerboeck P. \\
\hline MLC Component Output Block Length & 5 \\
\hline No. of LDPC Iterations & 3 \\
\hline LDPC Column Weight & 1 bit-per-channel-use \\
\hline Overall System Throughput & \\
\hline
\end{tabular}

TABLE II

SYSTEM PARAMETERS

the SP-demodulator has been updated with the extrinsic information received from all MSD levels.

The max-log approximation of the extrinsic LLR of a single bit $b_{k}$ output by the demodulator can be expressed as

$$
\begin{aligned}
& L\left(b_{k} \mid \tilde{x}\right)-L_{a}\left(b_{k}\right) \\
= & \max _{\mathbf{s}^{l} \in S_{1}^{k}}\left[-\frac{1}{2 \sigma_{w}^{2}}\left(\tilde{\mathbf{x}}-\alpha \cdot \mathbf{s}^{l}\right)\left(\tilde{\mathbf{x}}-\alpha \cdot \mathbf{s}^{l}\right)^{T}+\sum_{j=0, j \neq k}^{B-1} b_{j} L_{a}\left(b_{j}\right)\right] \\
- & \max _{\mathbf{s}^{l} \in S_{0}^{k}}\left[-\frac{1}{2 \sigma_{w}^{2}}\left(\tilde{\mathbf{x}}-\alpha \cdot \mathbf{s}^{l}\right)\left(\tilde{\mathbf{x}}-\alpha \cdot \mathbf{s}^{l}\right)^{T}+\sum_{j=0, j \neq k}^{B-1} b_{j} L_{a}\left(b_{j}\right)\right],
\end{aligned}
$$

where the SP symbols carry $B$ number of MLC bits, $\mathbf{b}=$ $b_{0}, \ldots, b_{B-1} \in\{0,1\}$. Let us assume furthermore that $S_{1}^{k}$ and $S_{0}^{k}$ represent two specific 2D subsets of the 4D SP symbol constellation $S$, which obey $S_{1}^{k} \triangleq\left\{\mathbf{s}^{l} \in S: b_{k}=1\right\}$ and $S_{0}^{k} \triangleq\left\{\mathbf{s}^{l} \in S: b_{k}=\right.$ $0\}$, respectively.

In general, for a MLC scheme having $q$ protection levels, the MLCencoded bits are mapped to a total of $N=2^{q}$ possible SP symbols. The updated a priori LLRs obtained from the preceding MLC protectionlevel at level $i=1 \ldots 4$ are given by $L_{C i}^{e} \triangleq\left\{L_{a}\left(b_{k}\right) ; k \in\{t q+(i-\right.$ $1), t=0,1, \ldots, N\}\}$.

The coding rates determined from the equivalent capacity rules detailed in [14] are shown in Table I. Table I shows the code rates of the LDPC component codes used for the SP-aided system as well as the benchmarker scheme employing QPSK. Note that the code rates are selected so that the SP-aided system and the QPSK one have an equivalent spectral efficiency of 1 bit-per-channel-use .

\section{Performance Results}

In this section, we consider the downlink (DL) of a generalised MC DS-CDMA system employing an $(M \times L)$-dimensional transmit AA in conjunction with $M=2, L=2$ and a single receive antenna, while using the coherent SSTS-SP in order to demonstrate the performance improvements achieved by the proposed system. Furthermore, all results were recorded for the coherent SSTS scheme assuming perfect channel knowledge at the receiver. All simulation parameters are listed in Table II.

In Figure 2 we plot the BER performance versus the $E_{b} / N_{0}$ of the SSTS-SP-aided generalised MC DS-CDMA DL scheme using a $(2 \times 2)$-dimensional AA $(M=2, L=2)$ for $K=1,4$ and 8 users, where $E_{b}$ denotes the energy per bit and $N_{0}$ denotes the double

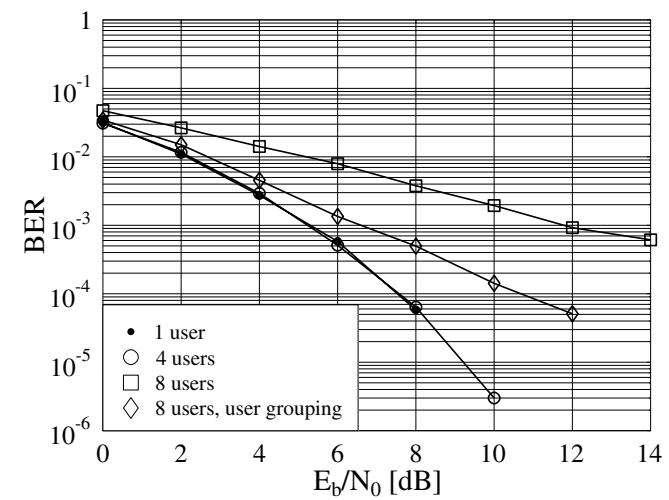

Fig. 2. BER performance of the SSTS-SP-aided generalised MC DS-CDMA DL using a $(2 \times 2)$-dimensional AA in conjunction with a varying number of users and the remaining system parameters outlined in Table II. Both TFdomain spreading and user grouping were employed to extend the capacity of the system, while supressing the MUI.

sided noise power spectral density. The remaining system parameters are outlined in Table II. Figure 2 shows that the performance of the system supporting $K=4$ users is similar to that of the system serving a single user, since no interference is encountered by the $K=4$ users employing different orthogonal 4-chip Walsh codes as their T-domain DS spreading in the synchronous DL. Let us now consider TF-domain spreading, which is employed for the sake of supporting $K=8$ users. Consequently, MUI is inevitably introduced among the users sharing the same T-domain spreading code. This becomes clear in Figure 2 for the case of $K=8$ users when no user grouping was employed, since the performance of the SSTS system supporting $K=8$ users is significantly worse than that supporting a single user or even $K=4$ users. However, when user grouping is employed by the SSTS system for the sake of reducing the MUI imposed, the performance of the SSTS-SP system supporting $K=8$ users substantially improves.

Figure 3 compares the attainable performance of the proposed $1 / 2$ rate MLC SSTS-SP-aided generalised MC DS-CDMA DL employing the code components of Table I and that of an identical-throughput 1 bit-per-channel-use uncoded SSTS-SP scheme using $L=4$. More explicitly, the effective throughput of 1 bit-per-channel-use of the SSTS-SP-MLC scheme is achieved by using a SP contellation supporting 4 bits-per-symbol with a $1 / 2$-rate SSTS and a $1 / 2$ rate MLC. Figure 3 shows the performance of the system, while employing $K=4$ user and the system parameters outlined in Table II. Observe in the figure that the system achieves a substantial performance improvement in conjunction with MLC decoding. However, we notice in Figure 3 that after two iterations, no more gain is attained. Explicitly, the figure demonstrates that a coding advantage of about $7.5 \mathrm{~dB}$ was achieved at a BER of $10^{-5}$ after two iterations by the $1 / 2$-rate MLC-coded SSTS-SP-aided generalised MC DSCDMA DL system over the identical-throughput uncoded SSTSSP scheme. Additionally, Figure 4 shows the effect of increasing the number of users on the MLC-coded SSTS-SP system, while employing TF-domain spreading both with and without the usergrouping technique. Similarly to Figure 2, Figure 4 shows that the performance of the system supporting $K=4$ users is reminiscent of that of the system serving a single user. Consequently, when TFdomain spreading is employed, MUI is inevitably introduced among the users sharing the same T-domain spreading code. This becomes clear for the case of $K=8$ users, when no user grouping was employed, since the performance of the SSTS system supporting $K=8$ users is significantly worse than that supporting a single user 


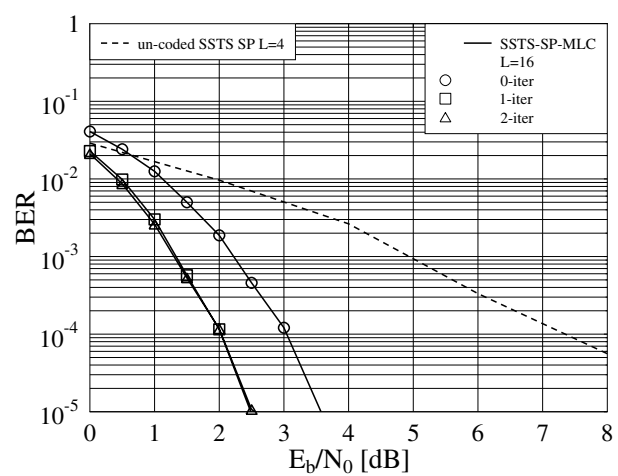

Fig. 3. BER performance of the SSTS-SP-aided generalised MC DS-CDMA DL using a $(2 \times 2)$-dimensional AA in conjunction with a $1 / 2$-rate MLC having the code rates of Table I and the system parameters outlined in Table II. The effective user throughput is 1 bit-per-channel-use and 4 users are supported using a spreading factor of 4 in both the T- and F-domain.

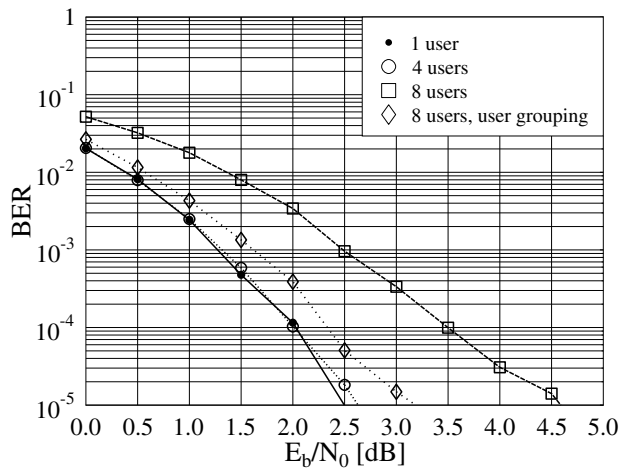

Fig. 4. BER performance of the SSTS-SP-aided generalised MC DS-CDMA DL using a $(2 \times 2)$-dimensional AA in conjunction with a $1 / 2$-rate MLC with the code rates of Table I and the system parameters outlined in Table II. The effective user throughput is 1 bit-per-channel-use, while using a spreading factor of 4 in both the T- and F-domain.

or even $K=4$ users. However, when the proposed user grouping is employed by the SSTS system for the sake of reducing the MUI imposed, the performance of the SSTS-SP system supporting $K=8$ users substantially improves.

Finally, Figure 5 compares the attainable BER performance of the proposed SSTS-SP-MLC scheme to that of the equivalent 1 bitper-channel-use throughput SSTS-MLC benchmarker employing the QPSK scheme of Table II. The BER curve of the proposed SSTS-SPMLC dips below $10^{-5}$ using a single iteration at $E_{b} / N_{0}=2.5 \mathrm{~dB}$. Upon employing $I=6$ iterations, the multidimensional SP aided system outperforms the conventional QPSK-aided system by an $E_{b} / N_{0}$ value of $3.8 \mathrm{~dB}$ at a BER of $10^{-5}$.

\section{CONCLUSION}

In this paper we proposed a novel system that amalgamates the advantages of spatial diversity, frequency diversity as well as beamforming gain based on the principle of SSTS in order to enhance the attainable performance of the generalised MC DSCDMA downlink, when operating in rapidly fading channels. The proposed system employs both time and frequency (TF) domain spreading for extending the capacity of the system, which is combined with a novel user-grouping technique for reducing the effects of Multiuser Interference (MUI). The SSTS system is combined with MLC and multidimensional SP modulation in order to enhance the attainable system performance as compared to the SSTS system employing conventional QPSK. Explicitly, our results demonstrate an approximately $3.8 \mathrm{~dB} E_{b} / N_{0}$ gain at a BER of $10^{-5}$ over an

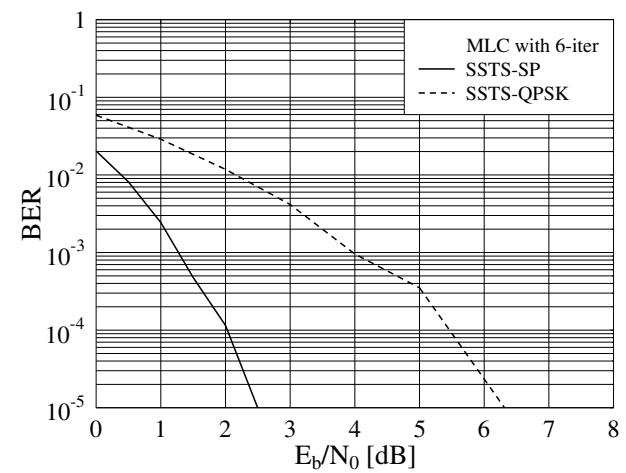

Fig. 5. BER performance Comparison of the SSTS-SP- and SSTS-QPSKaided generalised MC DS-CDMA DL using a $(2 \times 2)$-dimensional AA in conjunction with a $1 / 2$-rate MLC with the code rates of Table I and the system parameters outlined in Table II.

identical throughput 1 bit-per-channel-use scheme dispensing with SP modulation.

\section{REFERENCES}

[1] S. Alamouti, "A simple transmit diversity technique for wireless communications," IEEE Journal on Selected Areas in Communications, vol. 16, no. 8, pp. 1451-1458, 1998.

[2] V. Tarokh, H. Jafarkhani, and A. Calderbank, "Space-time block codes from orthogonal designs," IEEE Transactions on Information Theory, vol. 45, no. 5, pp. 1456-1467, 1999.

[3] B. Hochwald, T. Marzetta, and C. Papadias, "A transmitter diversity scheme for wideband CDMA systems based on space-time spreading," IEEE Journal on Selected Areas in Communications, vol. 19, no. 1, pp. 48-60, 2001.

[4] L. Hanzo, L.-L. Yang, E.-L. Kaun, and K. Yen, Single and Multi-Carrier DS-CDMA: Multi-user Detection, Space-Time Spreading, Synchronisation, Networking and Standards. Chichester, England: John Wiley and Sons Ltd and IEEE Press, NY, USA, 2003.

[5] E. A. Sourour and M. Nakagawa, "Performance of orthogonal multicarrier CDMA in a multipath fading channel," IEEE Transactions on Communications, vol. 44, pp. 356-367, Mar. 1996.

[6] L. Vandendorpe, "Multitone spread spectrum multiple access communications system in a multipath Rician fading channel," IEEE Transactions on Vehicular Technology, vol. 44, pp. 327-337, May 1995.

[7] L.-L. Yang and L. Hanzo, "Performance of broadband multicarrier DSCDMA using space-time spreading-assisted transmit diversity," IEEE Transactions on Wireless Communications, vol. 4, no. 3, pp. 885-894, 2005.

[8] L.-L. Yang and L. Hanzo, "Performance of generalized multicarrier DS-CDMA over Nakagami-m fading channels," IEEE Transactions on Communications, vol. 50, pp. 956-966, June 2002.

[9] J. Blogh and L. Hanzo, Third-generation systems and intelligent wireless networking: smart antennas and adaptive modulation. John Wiley \& Sons - IEEE Press, 2002.

[10] R. A. Soni, R. M. Buehrer, and R. D. Benning, "Intelligent antenna system for CDMA2000," IEEE Signal Processing Magazine, vol. 19, pp. 54-67, July 2002.

[11] W. Su, Z. Safar, and K. Liu, "Space-time signal design for timecorrelated Rayleigh fading channels," in IEEE International Conference on communications, vol. 5, pp. 3175-3179, 2003.

[12] O. Alamri, B. L. Yeap, and L. Hanzo, "A Turbo Detection and Sphere Packing Modulation Aided Space-Time Coding Scheme," IEEE Transactions on Vehicular Technology, vol. 56, pp. 575 - 582, March 2007.

[13] H. Imai and S. Hirakawa, "A new multilevel coding method using errorcorrecting codes," IEEE Transaction on Information Theory, vol. 23, pp. 371-377, May 1977.

[14] U. Wachsmann, R. F. H. Fischer, and J. B. Huber, "Multilevel codes: theoretical concepts and practical design rules," IEEE Transaction on Information Theory, vol. 45, pp. 1361-1391, July 1999.

[15] R. Gallager, Information Theory and Reliable Communication. New York: Wiley, 1968.

[16] J. H. Conway and N. J. Sloane, Sphere Packings, Lattices and Groups. Springer-Verlag, 1999. 